\title{
ARBORIZAÇÃO URBANA E SUSTENTABILIDADE: UMA PROPOSTA PARA O CENTRO EXPERIMENTAL DE TECNOLOGIAS HABITACIONAIS SUSTENTÁVEIS (CETHS)
}

\author{
Sérgio L. V. Tomasini ${ }^{1}$, Miguel Aloysio Sattler ${ }^{2}$
}

\section{RESUMO}

O Centro Experimental de Tecnologias Habitacionais Sustentáveis (CETHS) propôs um assentamento urbano experimental, destinado à população de baixa renda, localizado na área metropolitana de Porto Alegre. O projeto especifica novas tecnologias vinculadas a habitações sustentáveis, incorporando, por um lado, os preceitos do desenvolvimento sustentável e, por outro, a idéia de habitação como extensão do habitat do homem, considerando também o seu contexto social. Desta forma, integra também uma proposta de paisagismo, procurando agregar o maior número de funções possíveis à vegetação nesse espaço. Para tanto, foi elaborada uma pesquisa com o objetivo de produzir informações capazes de orientar especificamente o projeto de arborização urbana do loteamento. A seleção das espécies recomendadas partiu da listagem de espécies apresentada pelo Plano Diretor de Arborização Urbana de Porto Alegre e de cruzamento de informações de revisões bibliográficas, onde chegou-se à indicação de 72 espécies arbóreas, pertencentes a 29 famílias botânicas, todas pertencentes à região. Espera-se, portanto, que este trabalho possa, servir de subsídio e de incentivo para a comunidade que irá habitar o CETHS, no sentido de que as pessoas efetivamente conheçam, respeitem e usufruam das potencialidades das espécies que irão fazer parte do entorno de suas habitações.

Palavras-chave: paisagismo, arborização

\footnotetext{
. Engenheiro Agrônomo, Mestrando da UFRGS, Santa Maria/RS, sergiovtomasini@yahoo.com.br; 2. Engenheiro Civil e Agrônomo, PhD. Núcleo Orientado para a Inovação da Edificação - NORIE / UFRGS, sattler@vortex.ufrgs.br.
} 


\title{
URBAN ARBORIZATION AND SUSTENTABILIDADE: A PROPOSAL FOR THE EXPERIMENTAL CENTER OF SUSTAINABLE TECHNOLOGIES HABITACIONAIS (CETHS)
}

\begin{abstract}
The Experimental Center of Tecnologias Habitacionais Sustentáveis (CETHS) considered an experimental urban nesting, destined to the population of low income, located in the area metropolitan of Porto Alegre. The project specifies new entailed technologies the sustainable habitations, incorporating, the rules of sustainable development $\mathrm{e}$ the habitation idea as extension of the habitat of the man, also considering its social context. It also integrates a paisagismo proposal, looking for to add the biggest number of possible functions to the vegetation in this space. The research was elaborated to produce information capable to guide the project of urban arborization of the land division. The election of the recommended species left of the listing of species presented for the Managing Plan of Urban Arborization of Porto Alegre and crossing of information of bibliographical revisions, where it was arrived the indication of 72 arbóreas species, pertaining the 29 botanical families. One expects, therefore, that this work can, to serve of subsidy and incentive for the community that will go to inhabit the CETHS, in the direction of that the people effectively know, they respect and they usufruct of the potentialities of the species that will go to be part of entorno of its habitations.
\end{abstract}

Key-words: lanscape, arborization 


\section{INTRODUÇÃO}

O Centro Experimental de Tecnologias Habitacionais Sustentáveis (CETHS) propõe um assentamento urbano experimental, destinado à população de baixa renda. Localizado na área metropolitana de Porto Alegre, ele resulta de um projeto envolvendo a parceria entre o Núcleo Orientado para a Inovação da Edificação (NORIE / UFRGS) e a Prefeitura Municipal de Nova Hartz.

O projeto especifica novas tecnologias vinculadas a habitações sustentáveis, incorporando, por um lado, os preceitos do desenvolvimento sustentável e, por outro, a idéia de habitação como extensão do habitat do homem, considerando também o seu contexto social. O projeto foi desenvolvido a partir de uma série de intervenções sobre um loteamento convencional, que já contava com aprovação e financiamento da Secretaria Estadual de Habitação do RS, visando torná-lo mais sustentável. Dentre as estratégias empregadas no projeto neste sentido (como o uso de materiais construtivos de baixo impacto ambiental, tratamento local e reutilização dos resíduos domiciliares, captação e aproveitamento da água das chuvas, uso de fontes alternativas de energia, etc.), também houve a preocupação em desenvolver uma proposta paisagística para o loteamento, em harmonia com os preceitos de sustentabilidade, que regem o projeto como um todo. Desta forma, o paisagismo do CETHS está integrado a uma proposta que procura viabilizar a produção local de alimentos no loteamento.

O sistema de produção de alimentos, proposto pelo projeto, pode ser apresentado em dois níveis: - produção individual, nos lotes, e produção coletiva, em áreas de uso comum. Espera-se que, a partir da produção obtida nestes espaços, os moradores tenham condições de satisfazer parte de suas necessidades alimentares, bem como de dispor de uma fonte complementar de renda, através da comercialização dos excedentes. Embora existam locais específicos de produção previstos no projeto, como as áreas dos lotes e as de produção coletiva, tanto estas áreas desempenham outras funções no projeto global do CETHS, como outras áreas apóiam as funções de produção destas primeiras, sempre que possível. Visa-se aqui uma concepção holística de projeto e a convergência com dois princípios básicos da permacultura : 1) cada elemento do sistema deve executar diversas funções; 2) cada função importante é apoiada por muitos elementos (MOLLISON \& SLAY, 1998). Desta forma, a produção de alimentos em todo o projeto está intimamente associada ao paisagismo do loteamento e vice-versa.

Visando, portanto, agregar o maior número de funções possível à vegetação, bem como articulá-la com os demais elementos do projeto CETHS, a proposta de paisagismo para o loteamento procura atingir os seguintes objetivos: paisagismo produtivo, procurando, sempre que possível, utilizar espécies com características produtivas para o homem, ou 
interessantes para o ecossistema regional; paisagismo pedagógico, procurando fazer da arborização uma experiência de educação ambiental junto à comunidade; conforto ambiental, buscando a integração das árvores com o espaço construído, melhorando suas condições de conforto; conforto psicológico, procurando conferir à paisagem do CETHS valores estéticos que transmitam bem-estar físico e mental aos seus usuários.

"Permacultura é um sistema de design para a criação de ambientes humanos sustentáveis". "Em um primeiro nível, lida com as plantas, animais, edificações e infra-estruturas (água, energia, comunicações). Todavia a Permacultura não trata somente desses elementos, mas, principalmente, dos relacionamentos que podemos criar entre eles por meio da forma em que os colocamos no terreno" (MOLLISON \& SLAY, 1998).

Dentre as diversas questões envolvidas no paisagismo do CETHS, procurando cumprir as diretrizes anteriormente apresentadas, foi elaborada uma pesquisa com o objetivo de produzir informações capazes de orientar especificamente o projeto de arborização urbana do loteamento. Este estudo, cuja metodologia e resultados serão apresentados a seguir, servirá de base para o trabalho de eleição final das espécies a serem implantadas na arborização do loteamento e que será desenvolvido pelos técnicos da prefeitura de Nova Hartz, junto à comunidade que habitará o CETHS.

\section{MATERIAIS E MÉTODOS}

A relação de espécies que será utilizada como referência para a arborização do CETHS foi estabelecida a partir da compilação de uma série de dados obtidos através de revisão bibliográfica. A seleção das espécies recomendadas partiu da listagem de espécies apresentada pelo Plano Diretor de Arborização Urbana de Porto Alegre, como prioritária para a produção para fins de programas de arborização urbana nesse município (SANCHOTENE, 2000). Utilizou-se esta referência como ponto de partida, por entender-se que o conjunto de informações geradas pelo trabalho desenvolvido em Porto Alegre representa, atualmente, o material mais consistente envolvendo experiências com arborização urbana à nível de estado, sendo utilizado, inclusive, como referência para outras regiões do país.

A partir desta listagem inicial, realizou-se um cruzamento de informações com o Projeto Madeira do Rio Grande do Sul (REITZ et al, 1983), a fim de se verificar a origem das espécies listadas, selecionando-se, por fim, apenas as espécies de ocorrência natural neste estado. A fim de verificar, ainda quais dessas espécies são nativas para a região do Vale do Rio dos Sinos, da qual faz parte o Município de Nova Hartz, foram consultadas duas dissertações de mestrado realizadas junto ao Programa de Pós-Graduação em Botânica da 
UFRGS e que se dedicaram ao levantamento de comunidades arbóreas em distintas áreas dessa região do estado (DANIEL, 1992; ROSA, 1997).

Outras características foram levantadas sobre as espécies pesquisadas, a partir da consulta a diversas fontes bibliográficas (FRANCO, 199_; LONGHI, 1995; LORENZI, 1992; LORENZI, 1995; REITZ et al., 1983; ROSA, 1997; SANCHOTENE, 1989). São elas: nome comum, nome científico, possibilidades de aproveitamento (de acordo com os objetivos do CETHS) e fenologia (persistência foliar, período de floração e período de frutificação).

\section{RESULTADOS E DISCUSSÃO}

Através do cruzamento de informações, anteriormente referido, chegou-se à indicação de 72 espécies arbóreas, pertencentes a 29 famílias botânicas. Estas espécies foram agrupados em três tabelas distintas, relativas aos seus portes, que serão apresentadas a seguir, conforme a classificação apresentada pelo Plano Diretor de Arborização Urbana de Porto Alegre - PDAU (SANCHOTENE, 2000): espécies arbóreas de pequeno porte (Tabela 1), espécies arbóreas de médio porte (Tabela 2) e espécies arbóreas de grande porte (Tabela 3).

Tabela 1: Espécies arbóreas de pequeno porte para arborização de vias públicas (Projeto CETHS).

\begin{tabular}{|c|c|c|c|c|c|c|c|}
\hline \multirow[t]{2}{*}{ Nome científico } & \multirow[t]{2}{*}{ Nome Popular } & \multirow[t]{2}{*}{ Família } & \multirow[t]{2}{*}{ Origem } & \multirow[t]{2}{*}{ Aproveitamento } & \multicolumn{3}{|c|}{ Fenologia } \\
\hline & & & & & Folhas & Flores & Frutos \\
\hline $\begin{array}{l}\text { Blepharocalix } \\
\text { suaveolens }\end{array}$ & Camboim & Myitaceae & RS & $\overline{A F}, \mathrm{ME}$ & $\mathrm{P}$ & Set a Nov & Mar a $\mathrm{Abr}$ \\
\hline Eugenia hiemalis & \begin{tabular}{|l|} 
Aperta-cu, \\
Guamririmrde- \\
folha-miúda \\
\end{tabular} & Myita & RS & $\mathrm{AF}$ & $\mathrm{P}$ & Abr a Jun & Jul a Nov \\
\hline Brunfeisia unifiora & $\begin{array}{l}\text { Primavera, } \\
\text { Manacá }\end{array}$ & Solanaceae & RS & $\mathrm{FO}, \mathrm{ME}$ & $P$ & Ago a Set & Fev a Mar \\
\hline Calb̈andra twedü & \begin{tabular}{|l} 
Caliandra, \\
Topete-de- \\
cardeal \\
\end{tabular} & Mimosaceae & RS, VS & FO & $\mathrm{P}$ & Set a Mar & - \\
\hline Dodonea viscosa & $\begin{array}{l}\text { Vassoura- } \\
\text { vermelha }\end{array}$ & Sapindaceae & RS, VS & $\overline{-}$ & $\overline{-}$ & $\overline{-}$ & $\overline{-}$ \\
\hline Eugenia unifiora & Pitangueira & Myit & RS, VS & $\begin{array}{c}\mathrm{AH}_{,} \mathrm{AF}_{1} \mathrm{AD}, \\
\mathrm{ME}, \mathrm{ML}\end{array}$ & $P$ & Set a Out & Nov a Jan \\
\hline Gomidesio palustris & $\begin{array}{l}\text { Guarririm, } \\
\text { Uvá-mole }\end{array}$ & Myitaceae & $\overline{R S}$ & $\mathrm{AH}, \mathrm{AF}, \mathrm{ML}$ & $P$ & Nor a Jan & Fev a Jan \\
\hline Myrciaria cuspidata & Carrboim & Myitaceae & RS & $\begin{array}{c}\mathrm{AH}, \mathrm{AF}, \mathrm{ME} \text { 。 } \\
\mathrm{ML}\end{array}$ & $P$ & Nor a Jan & Nov a Jan \\
\hline Myrciaria deljcatula & Camboim bala & Myitaceae & RS & $\mathrm{AH}, \mathrm{AF}$ & $\mathrm{P}$ & Out a Mai & Nov a Jun \\
\hline $\begin{array}{l}\text { Myrrininum } \\
\text { loranthoides }\end{array}$ & $\begin{array}{l}\text { Verdadeiro pau } \\
\text { ferro, Muita }\end{array}$ & Myitaceae & RS, VS & $\overline{-}$ & - & - & $\overline{-}$ \\
\hline Fsidium cattjeyanum & Araçá & Myitaceae & RS & $\begin{array}{c}\mathrm{AH}, \mathrm{AF}, \mathrm{AS}, \\
\mathrm{AD}, \mathrm{ME}, \mathrm{MEL}\end{array}$ & $P$ & Nov a Dez & $\begin{array}{l}\text { Fev em } \\
\text { diante }\end{array}$ \\
\hline $\begin{array}{l}\text { Tabebuia } \\
\text { chrysctricha }\end{array}$ & Ipê-amarelo & Bignoniaceae & RS & $\mathrm{FO}$ & C & Ago a Set & Set a Out \\
\hline Tabebuia umbel/ata & Ipê-darvárzea & Bignoniaceae & $\mathrm{RS}, \mathrm{VS}$ & FO & $\mathrm{C}$ & Set a Nov & Dez a Mar \\
\hline $\begin{array}{l}\text { Tibouchinia } \\
\text { sellowiana }\end{array}$ & $\begin{array}{l}\text { Quaresmeira } \\
\text { da-serra }\end{array}$ & Melastomaceae & RS & FO & $P$ & Abr a Mai & Jan a Fer \\
\hline
\end{tabular}


Tabela 2: Espécies arbóreas de médio porte para arborização de vias públicas (Projeto CETHS).

\begin{tabular}{|c|c|c|c|c|c|c|c|}
\hline \multirow[t]{2}{*}{ Nome cientifico } & \multirow[t]{2}{*}{ Nome Popular } & \multirow[t]{2}{*}{ Familia } & \multirow[t]{2}{*}{ Origem } & \multirow[t]{2}{*}{ Aproveitamento } & \multicolumn{3}{|c|}{ Fenologia } \\
\hline & & & & & Folhas & Flares & Frutos \\
\hline Allophyllus edullis & Chal-chal & Sapindaceae & RS, VS & $\begin{array}{c}\mathrm{AH}, \mathrm{AF}, \mathrm{AS}, \\
\mathrm{ML}\end{array}$ & $P$ & Set a Nov & Nov a Dez \\
\hline Bauhinia candicans & Pata-de-vaca & Caesalpinaceą & RS & $\mathrm{ME}, \mathrm{FO}$ & $\overline{S C}$ & Nov a Dez & Abr a Mai \\
\hline Britoa guazumaefolla & Sete-capces & Myltaceae & RS & $\mathrm{AH}, \mathrm{AF}, \mathrm{ME}$ & $\bar{P}$ & aut a Nov & Mar a Abr \\
\hline Butio capitata & Butiazeiro & Palmae & RS & $\begin{array}{c}\mathrm{AH}_{2}, \mathrm{AF}_{3} \mathrm{AD}, \\
\mathrm{ML}, \mathrm{ME}\end{array}$ & $P$ & Set a Jan & Dez a Mar \\
\hline $\begin{array}{l}\text { Campomanesia } \\
\text { monbea }\end{array}$ & $\begin{array}{l}\text { Guabiroba } \\
\text { folharmiúda }\end{array}$ & Myltaceae & RS, VS & $\mathrm{AH}, \mathrm{UAF}$ & $P$ & Ago a Dez & Dez a Jan \\
\hline Casearia parvifilora & \begin{tabular}{|l|} 
Guassatunga \\
\end{tabular} & Flacouiticaceas & RS & $\mathrm{AF}, \mathrm{ME}$ & $\mathrm{P}$ & Set a Out & Set a Dez \\
\hline Erythrina crista-galli & \begin{tabular}{|l|} 
Corticeira-do- \\
banhado \\
\end{tabular} & Papilionaceae & RS, VS & FO & $\bar{C}$ & Set a Dez & Jan a Fev \\
\hline $\begin{array}{l}\text { Erythroxyl/um } \\
\text { argentinum }\end{array}$ & Cocão & \begin{tabular}{|c|}
$\begin{array}{c}\text { Erythroxylacea } \\
e\end{array}$ \\
\end{tabular} & RS, VS & $\mathrm{AF}, \mathrm{ME}, \mathrm{ML}$ & $P$ & Set a Out & Fev a Mar \\
\hline Eugenia pyniformis & Uvaia & Myitaceae & RS & $\mathrm{AH}, \mathrm{AF}_{1}, \mathrm{ME}$ & $P$ & Nor a Jan & Jan a Fev \\
\hline Eugenia shuechiana & Ástria & Myltaceae & RS, VS & $\mathrm{AH}, \mathrm{AF}$ & $\mathrm{P}$ & Ago & Set a Nov \\
\hline Myrcia multificra & $\begin{array}{l}\text { Camboimrda- } \\
\text { follharlarga }\end{array}$ & Myltaceae & RS, VS & - & - & - & - \\
\hline Rheedia gardneriana & Bacopari & Guttiferae & RS & $\begin{array}{c}\mathrm{AH}, \mathrm{AF}_{3} \mathrm{AS}, \\
\mathrm{ME}\end{array}$ & $\mathrm{P}$ & Ago a Set & Dez a Fev \\
\hline Rollinia exabida & Araticum & Annonaceae & RS, VS & $\mathrm{AH}, \mathrm{AF}, \mathrm{ME}$ & $\mathrm{C}$ & aut a Nov & Mar a Mai \\
\hline Schinus malle & \begin{tabular}{|l|} 
Aroeira- \\
periquito
\end{tabular} & Anacardiaceae & RS & $\mathrm{AF}, \mathrm{ME}, \mathrm{ML}$ & $\bar{P}$ & Ago a Nov & Dez a Jan \\
\hline Soroces bomplandij & Sincho & Moraceae & RS, VS & $\mathrm{AF}, \mathrm{ME}$ & $\mathrm{P}$ & Ago a Set & Fev a Mar \\
\hline Tabebuia puicherrima & Ipê- amarelo & Bignoniaceae & RS & $\mathrm{FO}$ & - & - & - \\
\hline Vitex montevidensis & Tarumã-preta & Verbenaceae & $\overline{R S}$ & $\begin{array}{c}\mathrm{AH}, \mathrm{AF}_{3} \mathrm{AS}, \\
\mathrm{ME}\end{array}$ & $\bar{C}$ & Out a Dez & Jan a Mar \\
\hline
\end{tabular}


Tabela 3: Espécies arbóreas de grande porte para arborização de vias públicas (Projeto CETHS).

\begin{tabular}{|c|c|c|c|c|c|c|c|}
\hline \multirow[t]{2}{*}{ Nome cientifico } & \multirow[t]{2}{*}{ Nome Popular } & \multirow[t]{2}{*}{ Familia } & \multirow[t]{2}{*}{ Origem } & \multirow[t]{2}{*}{ Aproveitamentr } & \multicolumn{3}{|c|}{ Fenologia } \\
\hline & & & & & Folhas & Flores & Frutos \\
\hline Apufeia lejocarpa & Grápia & Caesalpinace ae & RS & - & C & Set a Dez & Dez a Fev \\
\hline $\begin{array}{l}\text { Aspidosperma } \\
\text { arvifolium }\end{array}$ & Guatambú & Apocynaceae & RS & $\overline{-}$ & $\mathrm{SC}$ & Ago a Nov & Jul a Ago \\
\hline A tefeja glazioviana & Timbó & Fabaceae & RS & ME & $\mathrm{C}$ & Nov a Jan & Mar a Mai \\
\hline Banara parvifiora & Pau-vidro & Flacourtiace ae & RS & - & - & - & - \\
\hline Cabralea cangerana & Canjerana & Meliaceae & RS, VS & $\overline{A F}, \mathrm{ME}$ & $\bar{P}$ & $\begin{array}{l}\text { Set a Novt } \\
\text { Fev a Mar }\end{array}$ & $\begin{array}{l}\text { Julho em } \\
\text { diante }\end{array}$ \\
\hline $\begin{array}{l}\text { Campomanesia } \\
\text { anthocarpa }\end{array}$ & Guabirobeira & Myltaceae & RS, VS & $\begin{array}{c}\mathrm{AH}, \mathrm{AF}, \mathrm{AD}, \\
\mathrm{ME}\end{array}$ & $\mathrm{C}$ & Set a Out & Dez a Jan \\
\hline Cecropia catarinensis & Embaúba & Cecropiaceae & RS & $\mathrm{AF}, \mathrm{AS}, \mathrm{ME}$ & $\mathrm{P}$ & Dez a Mar & Abr a Jun \\
\hline Cedrefa fissilis & Cedro & Meliaceae & RS, VS & ME & $\mathrm{C}$ & Set a Dez & Jul a Ago \\
\hline Cordia trichotoma & Louro-branco & Boraginaceae & RS, VS & $\mathrm{FO}$ & $\mathrm{C}$ & Fev a $\mathrm{Abr}$ & Mai a Jul \\
\hline Cupania vernalis & $\begin{array}{l}\text { Camboatá } \\
\text { vermelho }\end{array}$ & Sapindaceae & RS, VS & $\mathrm{AF}, \mathrm{ML}$ & $\mathrm{P}$ & \begin{tabular}{|r|} 
Dez a Mar \\
Jun a Set
\end{tabular} & Out a Dez \\
\hline Eryithrina fakata & $\begin{array}{l}\text { Corticeira da } \\
\text { serra }\end{array}$ & Fabaceae & RS, VS & FO & SC & Out a Nov & Dez a Mar \\
\hline Eugenia involucrata & Cerejeira & Myltaceae & RS & $\begin{array}{c}\mathrm{AH}, \mathrm{AF}, \mathrm{AD}, \\
\mathrm{ME}\end{array}$ & $\mathrm{P}, \mathrm{SC}$ & Set a Nov & Out a Dez \\
\hline Eugenia rostrifolia & Batinga & Myitaceae & RS, VS & $\mathrm{AH}, \mathrm{AF}, \mathrm{AD}$ & $\mathrm{P}$ & Dez a Jan & Jun a Jul \\
\hline Guapira apositae & Maria mole & Nyctanginace ae & RS, VS & - & - & - & - \\
\hline Guarea lessoniana & $\begin{array}{l}\text { Camboatá- } \\
\text { branco }\end{array}$ & Meliaceae & RS & - & - & - & - \\
\hline Holocafyx balansae & Alecrim & Caesalpinaceae & RS & $\mathrm{ME}$ & $\mathrm{P}$ & Dez a Jan & Jun a Jul \\
\hline lex paraguaiensis & Erva-mate & Aquifoliace ae & RS, VS & $\mathrm{AH}, \mathrm{AF}, \mathrm{ME}$ & $\mathrm{P}$ & Set a Dez & Dez a Mar \\
\hline nga marginata & Ingá-feijão & Mimosaceae & RS, VS & $\begin{array}{c}\mathrm{AH}, \mathrm{AF}, \mathrm{AS}, \\
\mathrm{ML}\end{array}$ & $P$ & Out a Fev & Mar a Mai \\
\hline acaranda miorantha & Caroba & Bignoniaceae & RS, VS & $\mathrm{FO}, \mathrm{ME}$ & $\mathrm{C}$ & Out a Dez & Jul a Set \\
\hline Luhea divaricata & açoita-cavalo & Tiliaceae & RS, VS & $\mathrm{ME}, \mathrm{FO}$ & $\mathrm{C}$ & Dez a Fev & Mai a Jun \\
\hline Matayba efegnoides & $\begin{array}{l}\text { Camboatá- } \\
\text { branco }\end{array}$ & Sapindaceae & RS, VS & AF & $\mathrm{P}$ & Set a Nov & Dez a Jan \\
\hline Myrcia glabra & Uvá-duro & Mytaceae & RS & $\mathrm{AF}, \mathrm{FO}$ & $\mathrm{P}$ & Fev a $\mathrm{Abr}$ & Jun a Out \\
\hline Myrcianthes pungens & Guabïú & Myltaceae & RS, VS & $\begin{array}{c}\mathrm{AH}, \mathrm{AF}, \mathrm{AS}, \\
\mathrm{ME}, \mathrm{ML}\end{array}$ & $\mathrm{P}$ & Set a Nov & Jan a Mar \\
\hline Myrciaria truncifiora & Jaboticabeira & Myitaceae & RS & $\mathrm{AH}, \mathrm{AF}, \mathrm{ME}$ & $\mathrm{P}$ & Set a Out & Out a Nov \\
\hline Myrocarpus frondosus & Cabreúva & Fabaceae & RS, VS & ME, ML & $\mathrm{C}$ & Set a Out & Jan a Fev \\
\hline Ocotea puichella & Canela lajeana & Lauraceae & RS, VS & $A F$ & $\mathrm{P}$ & Nov a Jan & Mai a Jul \\
\hline Nectandra fanceolata & Canela & Lauraceae & RS, VS & $\mathrm{AF}$ & $\mathrm{P}$ & Nov a Jan & Mai a Jul \\
\hline $\begin{array}{l}\text { Nectandra } \\
\text { megapotamica }\end{array}$ & Canela-preta & Lauraceae & RS, VS & $A F$ & $\mathrm{P}$ & Ago a Out & Dez a $\mathrm{Abr}$ \\
\hline Parapiptadania rigida & $\begin{array}{l}\text { Angico- } \\
\text { vermelho }\end{array}$ & Mimosace ae & RS, VS & ME, ML & C & Nov a Dez & Jun a Jul \\
\hline Patagonuda americana & Guajuvira & Boraginaceae & RS, VS & ME & $\mathrm{C}$ & Set a Nov & Nov a Jan \\
\hline Peitophorum dubium & Canafistula & Caesalpinaceae & RS & $\mathrm{FO}$ & C & Nov a Fev & Mai a Jul \\
\hline Pinocarpus pinnabifolius & Jaborandi & Rutaceae & RS & $\mathrm{ME}, \mathrm{FO}$ & $\mathrm{P}$ & todo 0 and & todo 0 and \\
\hline Pouteria gardneriana & Aquaí & Sapotaceae & RS & - & - & - & - \\
\hline Pouteria salicifolia & $\begin{array}{l}\text { Aguai-folha-de } \\
\text { salso }\end{array}$ & Sapotaceae & RS, VS & - & - & - & - \\
\hline Rapanea um beflata & Capororoca & Myrsinaceae & RS & $\mathrm{AF}, \mathrm{ME}$ & $\mathrm{P}$ & Set a Dez & $\mathrm{Dez}$ a $\mathrm{Abr}$ \\
\hline Roupala brasilfensis & Carvalho-bras. & Proteaceae & RS & - & $\mathrm{C}$ & Jun a Ago & Ago a Out \\
\hline yagrus romanzoffiana & Gerivá & Palmae & RS, VS & $\begin{array}{c}\mathrm{AH}, \mathrm{AF}, \mathrm{AS}, \\
\mathrm{AD}, \mathrm{ML}\end{array}$ & $\mathrm{P}$ & Set a Mar & Fev a Ago \\
\hline Tabebuia alba & $\begin{array}{l}\text { Ipê-amarelo, } \\
\text { Ipê-ouro }\end{array}$ & Bignoniaceae & RS & $\mathrm{FO}$ & C & Jul a Set & Out a Nov \\
\hline Tabebuia avelfanedae & Ipê-rôxo & Bignoniaceae & RS, VS & $\mathrm{ME}, \mathrm{FO}$ & $\mathrm{C}$ & Set a Fev & Out a Mar \\
\hline Trema miqantha & Grandiuya & Ulmaceae & RS, VS & $\mathrm{AF}, \mathrm{AD}, \mathrm{ML}$ & $\mathrm{P}$ & Set a Jan & Jan a Mai \\
\hline Trichilia claussenü & Catiguá & Meliaceae & RS, VS & $A F$ & SC & Ago a Out & Jan a Mar \\
\hline
\end{tabular}

Origem: RS - nativa do Estado do Rio Grande do Sul; VS - nativa da região do Vale do Sinos (segundo ROSA, 1997 e DANIEL, 1992 ) Aproveitamento: AH - alimentação humana; AF alimento para a avifauna; AS - alimento para outros animais silvestres; AD - alimento para animais domésticos; ME - uso medicinal; ML - melífera Persistência foliar: $\mathrm{P}$ - persistente; SC - semi-caduca; C - caduca 


\section{CONCLUSÕES}

O levantamento realizado por este trabalho permitiu constatar a convergência entre a busca pela construção de um espaço urbano mais sustentável e a utilização de espécies nativas nos programas de arborização urbana. Além de incentivar o incremento da biodiversidade no ambiente construído e amenizar os impactos produzidos pelo o homem sobre os ecossistemas naturais, o uso de espécies nativas pode representar uma importante fonte de recursos para o homem.

A maior restrição à utilização destas espécies na arborização urbana ainda está, no entanto, relacionada à falta de conhecimento geral de sua importância para o meio ambiente e de seu potencial de utilização pelas populações urbanas. Esta preocupação constituiu a principal motivação que levou ao desenvolvimento deste trabalho. Espera-se, portanto, que este trabalho possa, de fato, servir de subsídio e de incentivo para a comunidade que irá habitar o CETHS, no sentido de que as pessoas efetivamente conheçam, respeitem e usufruam das potencialidades das espécies que irão fazer parte do entorno de suas habitações.

\section{REFERÊNCIAS BIBLIOGRÁFICAS}

DANIEL, A. Estudo fitossociológico arbóreo arbustivo da mata ripária da bacia hidrográfica do Rio dos Sinos, RS. Porto Alegre: UFRGS, 1992. Dissertação de Mestrado.

FRANCO, I.J. Ervas e plantas medicinais: a medicina dos simples. Chapecó: Imprimax, 199

LONGHI, R.A. Livro das árvores: árvores e arvoretas do Sul. Porto Alegre: L\&PM, 1995.

LORENZI, H. Árvores brasileiras: manual de identificação e cultivo de plantas arbóreas nativas do Brasil. Nova Odessa: Editora Plantarum, 1992.

LORENZI, H. Plantas ornamentais no Brasil: arbustivas, herbáceas e trepadeiras. Nova Odessa: Editora Plantarum, 1995.

MOLLISON, B. \& SLAY, R.M. Introdução à permacultura. Brasília: MA/SDR/PNF, 1998. 204 p. 
REITZ R; KLEIN, R.M.; REIS, A. Projeto madeira do Rio Grande do Sul. Itajaí: Herbário Barbosa Rodrigues, 1983.

ROSA, F.F. Contribuições ao conhecimento das comunidades vegetais da planície de inundação do Rio dos Sinos, RS. Porto Alegre: UFRGS, 1997. Dissertação de Mestrado.

SANCHOTENE, M.C.C. Fruteiras nativas úteis à fauna na arborização urbana. Porto Alegre: FEPLAM, 1989.

SANCHOTENE, M.C.C. (cord). Plano Diretor de Arborização de Vias Públicas. Porto Alegre: Secretaria Municipal do Meio Ambiente, 2000. 